Bull. Korean Math. Soc. 49 (2012), No. 6, pp. 1255-1262

http://dx.doi.org/10.4134/BKMS.2012.49.6.1255

\title{
GLOBAL ASYMPTOTIC STABILITY FOR A DIFFUSION LOTKA-VOLTERRA COMPETITION SYSTEM WITH TIME DELAYS
}

\author{
JiA-FAng Zhang and Ping-An Zhang
}

\begin{abstract}
A type of delayed Lotka-Volterra competition reaction-diffusion system is considered. By constructing a new Lyapunov function, we prove that the unique positive steady-state solution is globally asymptotically stable when interspecies competition is weaker than intraspecies competition. Moreover, we show that the stability property does not depend on the diffusion coefficients and time delays.
\end{abstract}

\section{Introduction}

In this paper we consider the following diffusion Lotka-Volterra competition system with two time delays

$$
\left\{\begin{array}{l}
\frac{\partial u(t, x)}{\partial t}=d_{1} \Delta u(t, x)+r_{1} u(t, x)\left[1-u(t, x)-a v\left(t-\tau_{1}, x\right)\right], x \in \Omega, t>0 \\
\frac{\partial v(t, x)}{\partial t}=d_{2} \Delta v(t, x)+r_{2} v(t, x)\left[1-b u\left(t-\tau_{2}, x\right)-v(t, x)\right], x \in \Omega, t>0 \\
\frac{\partial u}{\partial \nu}=\frac{\partial v}{\partial \nu}=0, x \in \partial \Omega, t \geq 0 \\
u(t, x)=\phi(t, x) \geq 0, v(t, x)=\psi(t, x) \geq 0,(t, x) \in[-\tau, 0] \times \Omega,
\end{array}\right.
$$

where the functions $u(t, x)$ and $v(t, x)$ stand for the two population densities; For $i=1,2$, let $d_{i}>0$, denote the diffusion coefficients of two species; $r_{i}>0$, denote the intrinsic growth rates; $a, b$ are competition coefficients between the species; $\tau_{i}$ are competition delays; $\Omega$ is a bounded domain in $\mathbb{R}^{n}$, with smooth boundary $\partial \Omega$; $\nu$ is the unit outward normal vector on the boundary of $\Omega$. The Neumann boundary condition implies that the two species have no flux across the boundary and the parameter $\tau=\tau_{1}+\tau_{2}$.

As mentioned by Faria and Oliveira in [3], the time-delays in differential equations arise naturally in mathematical models in biology, to account for the maturation period of biological species, synaptic transmission time among neurons, incubation time in epidemic models, and various other situations $[5,6]$. For a detailed discussion of this subject we refer to $[2,8,9,14,18,21]$.

Received January 14, 2011; Revised April 17, 2012.

2010 Mathematics Subject Classification. 35K57, 92D25.

Key words and phrases. global asymptotic stability, delays, Lyapunov function. 
Qualitative analysis of the systems like (1.1) has been investigated in many literatures (see $[1,11,15]$ ). Our model is a simplified diffusion version of the system discussed in [15, Chapter 5.7]. Similar to [15], the self-limitation or crowding effect in (1.1) is represented by an instantaneous term. The competition of population $u$ on $v$ is measured by competition coefficient $a$ with time delay, and $b$ has a similar meaning. In addition, the stability of constant steady states of the system (1.1) with distributed delays are also considered by some authors, see $[4,13]$.

Early stability discussion was always via the semigroup theory and the theory of dynamical systems (see $[10,20]$ and the references therein). More recently, the method of upper and lower solutions combined with their monotone iterations has also been used. As pointed out in [11], the determination of the precise asymptotic limit of the time-dependent solution is, in general, more difficult especially when the system possesses multiple steady-state solutions. Moreover, in our situation, the construction of proper upper and lower solutions is very tough, and this motivates us to find an alternative method.

The main subject of this paper is to investigate the stability of the positive steady-state solution of system (1.1). By constructing a new Lyapunov function and using Lyapunov's second method, we prove that the positive constant steady-state solution is globally asymptotically stable when interspecies competition is weaker than intraspecies competition. We hope that this result could provide another method in understanding the dynamics of the diffusion Lotka-Volterra competition system with two time delays. The main result and its proof are given in Section 2. Finally, in Section 3, we apply our results to a model investigated by $\mathrm{Pao}$ [11] and give some discussions and numerical simulations.

\section{Global stability of positive constant steady-state}

In this section, by using the Lypunov's second method, we mainly investigate the global asymptotic stability of positive constant steady-state when interspecies competition is weaker than intraspecies one $(0<a<1,0<b<1)$. It is easy to see that when $\frac{1-a}{1-a b}>0, \frac{1-b}{1-a b}>0$, the system (1.1) has a unique component positive constant steady-state solution $\left(u^{*}, v^{*}\right)$, where

$$
u^{*}=\frac{1-a}{1-a b}, \quad v^{*}=\frac{1-b}{1-a b} .
$$

The main theorem in this paper is the following:

Theorem 2.1. Suppose that $0<a<1,0<b<1$. Then the positive constant steady-state solution $\left(u^{*}, v^{*}\right)$ of $(1.1)$ is globally asymptotically stable for all nonnegative $\tau_{1}, \tau_{2}$, that is, $\left(u^{*}, v^{*}\right)$ attracts every solution of (1.1). 
Proof. According to [19], there exists a continuous function $g(x, t)$ on $\bar{\Omega} \times[0, \infty)$ such that (1.1) has the following form

$$
\begin{cases}\frac{\partial u(t, x)}{\partial t}=d_{1} \Delta u(t, x)+r_{1} u(t, x) g(x, t), & x \in \Omega, t>0, \\ \frac{\partial u}{\partial \nu}=0, & x \in \partial \Omega, t>0, \\ u(0, x)=u_{0}(x) \geq 0, & x \in \bar{\Omega} .\end{cases}
$$

From the maximum principle for scalar parabolic boundary value problems, we have that $u(t, x) \equiv 0$ on $[0, \infty) \times \bar{\Omega}$ if $u_{0}(x) \equiv 0$ in $\bar{\Omega}$, and $u(t, x)>0$ on $(0, \infty) \times \bar{\Omega}$ if $u_{0}(x)>0$ in $\bar{\Omega}$. Similarly, $v(t, x) \equiv 0$ on $[0, \infty) \times \bar{\Omega}$ if $v_{0}(x) \equiv 0$ in $\bar{\Omega}$, and $v(t, x)>0$ on $(0, \infty) \times \bar{\Omega}$ if $v_{0}(x) \not \equiv 0$ in $\bar{\Omega}$. In addition, by Proposition 4.2 of Chapter 9 in Wu [20] or Martin [10], the mild solution of (1.1) exists, and remains positive for all $t>0$ if $\phi(0, x)>0, \varphi(0, x)>0$ for $x \in \Omega$. Therefore, the solution $(u(t, x), v(t, x))$ is nonnegative.

Now, we define the following Lyapunov function

$$
\begin{aligned}
W(u, v)= & \int_{\Omega}\left\{\frac{1}{r_{1}}\left(u-u^{*}-u^{*} \ln \frac{u}{u^{*}}\right)+\frac{1}{r_{2}}\left(v-v^{*}-v^{*} \ln \frac{v}{v^{*}}\right)\right. \\
& \left.+\frac{1}{2} \int_{t-\tau_{2}}^{t}\left(u(\theta)-u^{*}\right)^{2} \mathrm{~d} \theta+\frac{1}{2} \int_{t-\tau_{1}}^{t}\left(v(\theta)-v^{*}\right)^{2} \mathrm{~d} \theta\right\} \mathrm{d} x .
\end{aligned}
$$

It is easy to check that $W(u, v)$ is nonnegative and $W(u, v)=0$ if and only if $u=u^{*}, v=v^{*}$.

Differentiating the Lyapunov function $W(u, v)$ with respect to time $t$ along the solutions of the system (1.1), we get

$$
\begin{aligned}
\frac{\mathrm{d} W(u, v)}{\mathrm{d} t}= & \int_{\Omega}\left\{\frac{u-u^{*}}{r_{1} u} u_{t}+\frac{1}{2}\left[\left(u-u^{*}\right)^{2}-\left(u\left(t-\tau_{2}\right)-u^{*}\right)^{2}\right]\right. \\
& \left.+\frac{v-v^{*}}{r_{2} v} v_{t}+\frac{1}{2}\left[\left(v-v^{*}\right)^{2}-\left(v\left(t-\tau_{1}\right)-v^{*}\right)^{2}\right]\right\} \mathrm{d} x \\
= & \int_{\Omega}\left\{\frac{u-u^{*}}{r_{1} u} d_{1} \triangle u-\left(u-u^{*}\right)\left[\left(u-u^{*}\right)+a\left(v\left(t-\tau_{1}\right)-v^{*}\right)\right]\right. \\
& +\frac{1}{2}\left[\left(u-u^{*}\right)^{2}-\left(u\left(t-\tau_{2}\right)-u^{*}\right)^{2}\right] \\
& +\frac{1}{2}\left[\left(v-v^{*}\right)^{2}-\left(v\left(t-\tau_{1}\right)-v^{*}\right)^{2}\right] \\
& \left.+\frac{v-v^{*}}{r_{2} v} d_{2} \triangle v-\left(v-v^{*}\right)\left[\left(v-v^{*}\right)+b\left(u\left(t-\tau_{2}\right)-u^{*}\right)\right]\right\} \mathrm{d} x .
\end{aligned}
$$

From the homogeneous Neumann condition and the Green's first identity, we get

and

$$
\int_{\Omega} \frac{u-u^{*}}{r_{1} u} d_{1} \triangle u \mathrm{~d} x=-\int_{\Omega} \frac{d_{1} u^{*}}{r_{1} u^{2}}|\nabla u|^{2} \mathrm{~d} x,
$$

$$
\int_{\Omega} \frac{v-v^{*}}{r_{2} v} d_{2} \triangle v \mathrm{~d} x=-\int_{\Omega} \frac{d_{2} v^{*}}{r_{2} v^{2}}|\nabla v|^{2} \mathrm{~d} x
$$


Therefore

$$
\begin{aligned}
\frac{\mathrm{d} W(u, v)}{\mathrm{d} t}= & \int_{\Omega}\left\{-\frac{d_{1} u^{*}}{r_{1} u^{2}}|\nabla u|^{2}-\frac{1}{2}\left[\left(u-u^{*}\right)+a\left(v\left(t-\tau_{1}\right)-v^{*}\right)\right]^{2}\right. \\
& -\frac{d_{2} v^{*}}{r_{2} v^{2}}|\nabla v|^{2}-\frac{1}{2}\left[\left(v-v^{*}\right)+b\left(u\left(t-\tau_{2}\right)-u^{*}\right)\right]^{2} \\
& -\frac{1}{2}\left(1-b^{2}\right)\left(u\left(t-\tau_{2}\right)-u^{*}\right)^{2} \\
& \left.-\frac{1}{2}\left(1-a^{2}\right)\left(v\left(t-\tau_{1}\right)-v^{*}\right)^{2}\right\} \mathrm{d} x
\end{aligned}
$$

It is easy to see that $0<a<1$ and $0<b<1$ imply $\frac{\mathrm{d} W(u, v)}{\mathrm{d} t}<0$.

In addition, using the comparison argument for parabolic problem, one can easily see that $0<u(t, x) \leq U(t, x)$ for all $(t, x) \in(0, \infty) \times \Omega$, where $U$ is the unique solution of

$$
\begin{cases}\frac{\partial U(t, x)}{\partial t}=d_{1} \triangle U(t, x)+r_{1} U(t, x)[1-U(t, x)], & x \in \Omega, t>0 \\ \frac{\partial u}{\partial \nu}=0, & x \in \partial \Omega, t>0 \\ u(0, x)=u_{0}(x) \geq 0, & x \in \Omega\end{cases}
$$

Then we can find a large $T$ such that $u(t, x) \leq 1+\epsilon$ in $[T, \infty) \times \Omega$ for any positive constant $\epsilon$ from the fact that $u(t, x) \leq U(t, x)$ and $U(t, x) \rightarrow 1$ as $t \rightarrow \infty$. Moreover, $v(t, x)$ has the same property. Therefore the solution $\left(u^{*}, v^{*}\right)$ is globally asymptotically stable. This completes the proof.

\section{Concluding remarks}

Generally, it has been recognized that the introduction of the time delays not only destabilizes the system but also could induce various oscillations and periodic solutions (see $[6,20]$ ). However, when the interspecies competition is weaker than intraspecies one, which is more natural in biology and ecology, the global asymptotic stability of the positive constant steady-state solution of (1.1) is found by using Lyapunov's second method in our work. This phenomenon implies that delays do not destabilize the system under the conditions $0<a, b<1$. We would also like to mention that turing instability has been proposed as a mechanism for pattern formation in numerous embryological and ecological contexts (see [17]). While, under our conditions, the possible instability phenomenon does not occur even with variable diffusion coefficients. Moreover, the stability of constant steady states of the system (1.1) with distributed delays had also been considered by some authors (see [4]).

The results in the literature [16] show that changes of hunting delays for system (1.1) without diffusions do not lead to the occurrence of Hopf bifurcation when interspecies competition is weaker than intraspecies competition. Our result here further indicates that the positive constant steady-state solution $\left(u^{*}, v^{*}\right)$ is globally asymptotically stable for any $d_{1}, d_{2}, \tau_{1} \geq 0, \tau_{2} \geq 0$, i.e., the positive constant steady-state solution cannot be destabilized by changing 
the diffusivity and time delays. The form of the Lyapunov function here is motivated by $[7,12]$. We notice that the result of Theorem 2.1 only depends on the parameters $a, b$.

As mentioned in the introduction, we consider an example from Pao [11]:

$$
\begin{cases}u_{t}-d_{1} \triangle u=u\left[a_{1}-b_{1} u-c_{1} J_{2} * v\right], & x \in \Omega, t>0, \\ v_{t}-d_{2} \triangle v=v\left[a_{2}-b_{2} J_{1} * u-c_{2} v\right], & x \in \Omega, t>0,\end{cases}
$$

where $a_{i}, b_{i}$ and $c_{i}, i=1,2$, are positive constants. $J_{2} * v, J_{1} * u$ are given by (1.2) of [11].

Letting $\bar{u}=\frac{b_{1}}{a_{1}} u, \bar{v}=\frac{c_{2}}{a_{2}} v$, and dropping the bars for simplification, (3.1) is transformed into

$$
\begin{cases}u_{t}-d_{1} \triangle u=a_{1} u\left[1-u-a J_{2} * v\right], & x \in \Omega, t>0, \\ v_{t}-d_{2} \triangle v=a_{2} v\left[1-b J_{1} * u-v\right], & x \in \Omega, t>0,\end{cases}
$$

where $a=\frac{a_{2} c_{1}}{a_{1} c_{2}}>0, b=\frac{a_{1} b_{2}}{a_{2} b_{1}}>0$. By using our new Lyapunov function, we could also obtain the global asymptotic stability of positive constant steadystate solution of system (3.1) when $a<1, b<1$.

The biological interpretation of Theorem 2.1 is that for a large type of competition systems, coexistence will always hold, which is a very common phenomena in the natural world.

In the following, we give some numerical simulations for a special case of system (1.1). We consider system (1.1) with the coefficients $r_{1}=r_{2}=1$, $a=0.8, b=0.5$. Obviously, $a<1, b<1$, therefore, system (1.1) has a unique positive constant steady-state $\left(\frac{1}{3}, \frac{5}{6}\right)$. From Theorem 2.1 , we know that the positive constant steady-state $\left(\frac{1}{3}, \frac{5}{6}\right)$ is globally asymptotically stable when $a<1, b<1$. The numerical simulations are shown in Figs. 1-4.
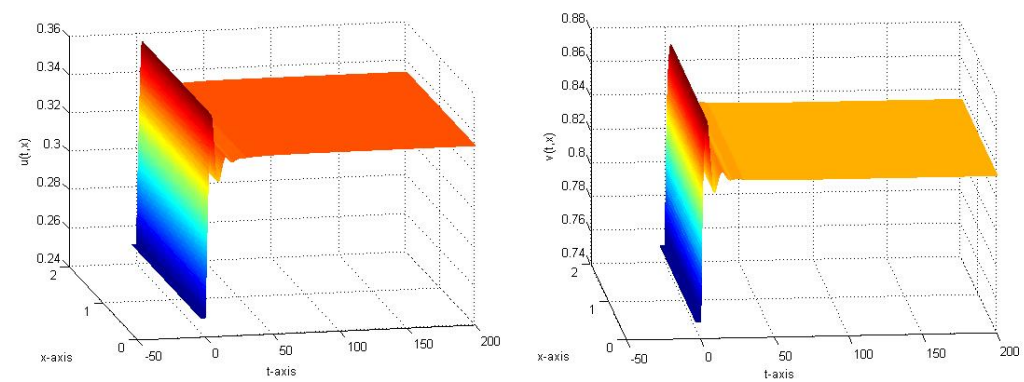

Figure 1. The numerical approximation to the solution of system (1.1) with $\tau_{1}=\tau_{2}=3.5, d_{1}=10, d_{2}=60$ and initial conditions $u(t, x)=0.25, u(t, x)=0.75$.

Acknowledgment. We would like to thank Professor Wan-Tong Li for many helpful discussions. The authors thank the referee for his/her careful reading 

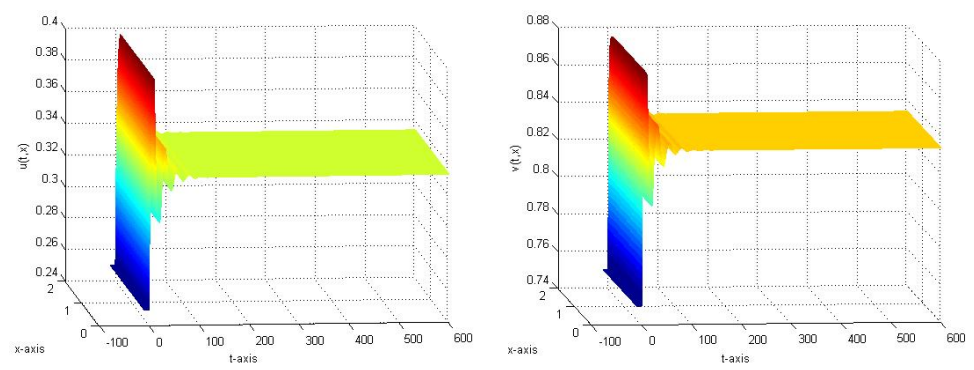

Figure 2. The numerical approximation to the solution of system (1.1) with $\tau_{1}=\tau_{2}=10, d_{1}=10, d_{2}=60$ and initial conditions $u(t, x)=0.25, u(t, x)=0.75$.
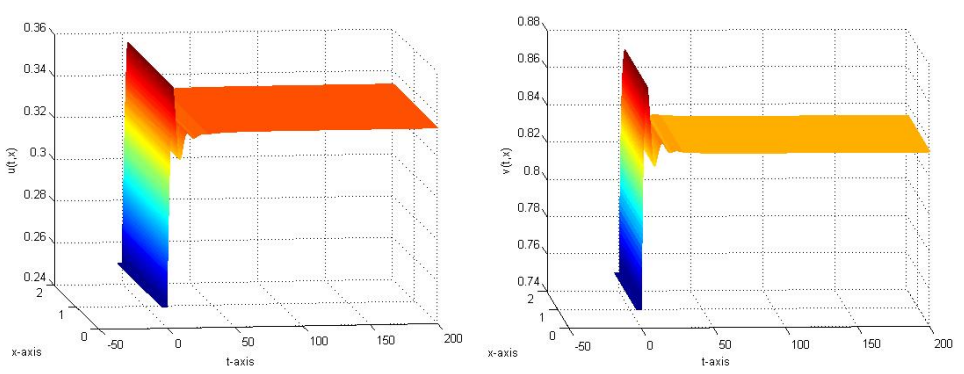

FiguRE 3. The numerical approximation to the solution of system (1.1) with $\tau_{1}=\tau_{2}=3.5, d_{1}=100, d_{2}=1$ and initial conditions $u(t, x)=0.25, u(t, x)=0.75$.
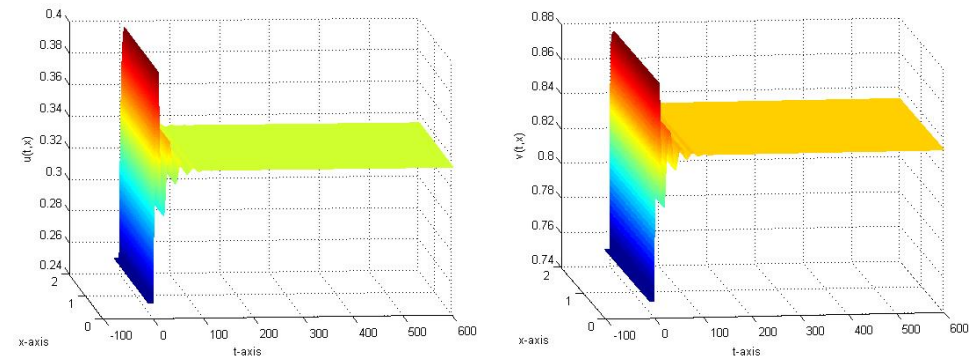

Figure 4. The numerical approximation to the solution of system (1.1) with $\tau_{1}=\tau_{2}=10, d_{1}=100, d_{2}=1$ and initial conditions $u(t, x)=0.25, u(t, x)=0.75$.

of the manuscript and valuable comments. The first author's research was partially supported by the Fundamental Research Fund of Henan University 
(B2011088). The second author's research was partially supported by the Fundamental Research Funds for the Central Universities (No. K5051270011).

\section{References}

[1] P. N. Brown, Decay to uniform states in ecological interaction, SIAM J. Appl. Math. 38 (1980), no. 1, 22-37.

[2] Y. H. Fan and L. L. Wang, Global asymptotical stability of a logistic model with feedback control, Nonlinear Anal. Real World Appl. 11 (2010), no. 4, 2686-2697.

[3] T. Faria and J. J. Oliveira, Local and global stability for Lotka-Volterra systems with distributed delays and instantaneous negative feedbacks, J. Differential Equations 244 (2008), no. 5, 1049-1079.

[4] S. Gourley and S. Ruan, Convergence and travelling fronts in functional differential equations with nonlocal term: a competition model, SIAM J. Math. Anal. 35 (2003), no. 3, 806-822.

[5] H. F. Huo and W. T. Li, Stable periodic solution of the discrete periodic Leslie-Gower predator-prey model, Math. Comput. Modelling 40 (2004), no. 3-4, 261-269.

[6] Y. Kuang, Delay Differential Equations with Application in Population Dynamics, Academic Press, New York, 1993.

[7] S. M. Lenhart and C. C. Travis, Global stability of a biological model with time delay, Proc. Amer. Math. Soc. 96 (1986), no. 1, 75-78.

[8] W. T. Li, G. Lin, and S. Ruan, Existence of travelling wave solutions in delayed reactiondiffusion systems with applications to diffusion-competition systems, Nonlinearity 19 (2006), no. 6, 1253-1273.

[9] Z. Ma, Mathematical Modeling and Research on the Population Ecology, AnHui educational press, Hefei, 1996

[10] R. H. Martin and H. L. Smith, Reaction-diffusion systems with time delays: monotonicity, invariance, comparison and convergence, J. Reine Angew. Math. 413 (1991), $1-35$.

[11] C. V. Pao, Convergence of solutions of reaction-diffusion systems with time delays, Nonlinear Anal. 48 (2002), no. 3, Ser. A: Theory Methods, 349-362.

[12] R. Peng and M. Wang, Note on a ratio-dependent predator-prey system with diffusion, Nonlinear Anal. Real World Appl. 7 (2006), no. 1, 1-11.

[13] S. Ruan and J. Wu, Reaction-diffusion equations with infinite delay, Canad. Appl. Math. Quart. 2 (1994), no. 4, 485-550.

[14] S. Ruan and X. Q. Zhao, Persistence and extinction in two species reaction-diffusion systems with delays, J. Differential Equations 156 (1999), no. 1, 71-92.

[15] H. L. Smith, Monotone Dynamical Systems: An Introduction to the Theory of Competitive and Cooperative Systems, Math. Surveys Monogr., vol. 41, American Mathematical Society, Providence, RI, 1995.

[16] Y. Song, M. Han, and Y. Peng, Stability and Hopf bifurcation in a competitive LotkaVolterra system with two delays, Chaos Solitons Fractals 22 (2004), no. 5, 1139-1148.

[17] A. M. Turing, The chemical basis of morphogenesis, Philos. Trans. R. Soc. Lond. B 237 (1952), 37-72.

[18] L. L. Wang and Y. H. Fan, Note on permanence and global stability in delayed ratiodependent predator-prey models with monotonic functional response, J. Comput. Appl. Math. 234 (2010), no. 2, 477-487.

[19] Y. M. Wang, Asymptotic behavior of solutions for a cooperation-diffusion model with a saturating interaction, Comput. Math. Appl. 52 (2006), no. 3-4, 339-350.

[20] J. Wu, Theory and Applications of Partial Functional Differential Equations, SpringerVerlag, New York, 1996. 
[21] J. Zhen and Z. Ma, Stability for a competitive Lotka-Volterra system with delays, Nonlinear Anal. 51 (2002), no. 7, 1131-1142.

JiA-FANG ZHANG

School of Mathematics and Information Sciences

HENAN UNIVERSITY

Kaifeng, Henan 475001, P. R. China

E-mail address: jfzhang@henu.edu.cn

Ping-An Zhang

Department of Mathematics

Xidian University

XI'AN, 710071, P. R. CHINA

E-mail address: pinganzhang@xidian.edu.cn 